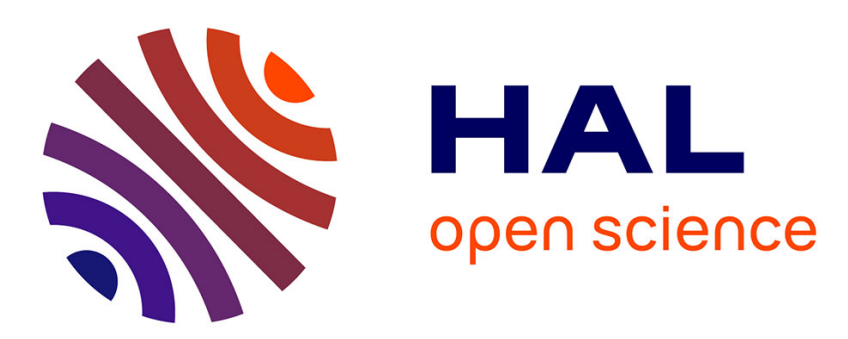

\title{
Optimal control problems of BV trajectories with pointwise state constraints
}

Nicolas Forcadel, Zhiping Rao, Hasnaa Zidani

\section{To cite this version:}

Nicolas Forcadel, Zhiping Rao, Hasnaa Zidani. Optimal control problems of BV trajectories with pointwise state constraints. 18th IFAC World Congress, Aug 2011, Milan, Italy. 10.3182/201108286-IT-1002.01694 . inria-00639021

\section{HAL Id: inria-00639021 \\ https://hal.inria.fr/inria-00639021}

Submitted on 7 Nov 2011

HAL is a multi-disciplinary open access archive for the deposit and dissemination of scientific research documents, whether they are published or not. The documents may come from teaching and research institutions in France or abroad, or from public or private research centers.
L'archive ouverte pluridisciplinaire HAL, est destinée au dépôt et à la diffusion de documents scientifiques de niveau recherche, publiés ou non, émanant des établissements d'enseignement et de recherche français ou étrangers, des laboratoires publics ou privés. 


\title{
Optimal control problems of BV trajectories with pointwise state constraints*
}

\author{
N. Forcadel ${ }^{\dagger} \quad$ Z. Rao $^{\ddagger} \quad$ H. Zidani ${ }^{\S}$
}

\begin{abstract}
This paper deals with some optimal control problems governed by ordinary differential equations with Radon measures as data and with pointwise state constraints. We study the properties of the value function and obtain its characterization by means of an auxiliary control problem of absolutely continuous trajectories. For this, we use some known techniques of reparametrization and graph completion.

We are also interested in the characterization of the value function as the unique constrained viscosity solution of a Hamilton-Jacobi equation with measurable time dependant Hamiltonians.
\end{abstract}

\section{Introduction}

In this paper, we study a state constrained optimal control problem with a Radon measure term in the dynamics using a Hamilton-Jacobi-Bellman approach.

Consider the following control system:

$$
\left\{\begin{array}{l}
d Y(t)=g_{0}(t, Y(t), a(t)) d t+\sum_{i=1}^{M} g_{i}(t, Y(t)) d \mu_{i} \quad t \in(\tau, T] \\
Y\left(\tau^{-}\right)=X
\end{array}\right.
$$

where $X \in \mathbb{R}^{n}, \tau \geq 0$, the measurable control $a:(0,+\infty) \rightarrow \mathbb{R}^{m}$ takes values in a compact set $A \subset \mathbb{R}^{m}$, and $\left\{\mu_{i}\right\}_{i=1, \cdots, M}$ is a given family of Radon measures. Let $\varphi: \mathbb{R}^{n} \rightarrow \mathbb{R}$ be a Lipschitz continuous function, and consider the following state-constrained control problem:

$$
v(\tau, x)=\inf _{a(\cdot) \in A}\left\{\varphi\left(Y_{X, \tau}^{a}(T)\right), Y_{X, \tau}^{a}(\cdot) \text { solution of }(1.1), Y_{X, \tau}^{a}(t) \in \mathcal{K} \text { for } t \in[\tau, T]\right\},
$$

where $\mathcal{K} \subset \mathbb{R}^{n}$ is a closed set of state constraints. Due to the presence of the Radon measures, the definition of solution for the state equation (1.1) is not classical. We refer to the definition introduced by Bressan and Rampazzo [5] and Dal Maso and Rampazzo [7] using the technique of graph completion. Formally by a suitable change of variable in both time and primitive of Radon measures, we can reduce (1.1) to a usual controlled ordinary differential equation with a measurable time-dependent dynamics which is called the reparameterized system. We will prove that the initial trajectory stays in $\mathcal{K}$ if and only if the reparameterized trajectory stays in $\mathcal{K}$ under suitable assumptions on the fields $g_{i}$.

The new value function associated with this reparameterized system is then characterized by a Hamilton-Jacobi-Bellman equation. But due to the double presence of an only time-measurable

*This work is partially supported by the European Union under the 7th Framework Programme «FP7-PEOPLE2010-ITN», Grant agreement number 264735-SADCO.

${ }^{\dagger}$ CEREMADE, University Paris-Dauphine, Place du Maréchal de Lattre de Tassigny, 75775 Paris cedex 16, France. forcadel@ceremade.dauphine.fr

$\ddagger$ UMA - ENSTA ParisTech, 32 Bd Victor, 75732 Paris Cx 15, France. Zhiping.Rao@ensta-paristech.fr

§Projet COMMANDS (ENSTA ParisTech \& Inria Saclay), 32 Bd Victor, 75732 Paris Cx 15 , France. Hasnaa.Zidani@ensta-paristech.fr 
Hamiltonian and state constraints, the definition of viscosity solution is not classical. We will then introduce a new definition of $L^{1}$-constrained viscosity solution for HJB equations on closed domains and with time-measurable Hamiltonians. Then, we will check that this definition is suitable to obtain a characterization of the value function.

Let us note that HJB equations on an open set with time-measurable Hamiltonians has been already studied by Ishii in [10]. On the other hand, state-constrained control problems with continuous Hamiltonians have been studied by many authors, see [12, 9, 4] and the references therein.

Notations. For each $r>0, x \in \mathbb{R}^{n}$, we denote by $B(x, r)$ the closed ball with center $x$ and radius $r$. For a function $f:[a, b] \rightarrow \mathbb{R}^{n}$ we denote by $V_{a}^{b}(f)$ the total variation of $f$ on $[a, b]$ and by $B V\left([0, T] ; \mathbb{R}^{n}\right)$ the set of functions $f:[0, T] \rightarrow \mathbb{R}^{n}$ with bounded total variation on $[0, T]$.

In the sequel, we also use the notations: $f\left(t^{+}\right):=\lim _{s \rightarrow t^{+}} f(s), f\left(t^{-}\right):=\lim _{s \rightarrow t^{-}} f(s)$ and $[f]_{t}:=$ $f\left(t^{+}\right)-f\left(t^{-}\right)$. And finally, we denote by $A C\left([0,1] ; \mathbb{R}^{n}\right)$ the set of absolutely continuous functions from $[0,1]$ to $\mathbb{R}^{n}$.

\section{Problem with discontinuous dynamics}

In this section, we recall the definition of solution for the state equation (1.1) introduced by Dal Maso and Rampazzo [7] and we recall the graph completion construction, which allows to obtain an auxiliary system with measurable dynamics.

In the sequel, we consider the following assumption on the admissible controls:

(Hco) The set of controls $\mathcal{A}:=L^{\infty}((0, T) ; A)$ where $A \subset \mathbb{R}^{m}$ is a compact set, $m \geq 1$.

\subsection{The state equation}

Let $T$ be a fixed final time, $X \in \mathcal{K}$ be an initial data. Let $\left\{\mu_{i}\right\}_{i=1, \ldots, M}$ be a family of Radon measures, and $a \in \mathcal{A}$ be an admissible control variable. We denote by $Y_{x, \tau}^{a}(t): \mathbb{R}^{+} \rightarrow \mathbb{R}^{n}$ the controlled trajectory solution of (1.1) in the sens of the Definition 2.1.

On the functions $g_{i}, i=0, \cdots, M$, we assume that:

$(\mathrm{Hg} 1) g_{0}(t, Y, a): \mathbb{R}^{+} \times \mathbb{R}^{n} \times A \rightarrow \mathbb{R}^{n}$ and $g_{i}(t, Y): \mathbb{R}^{+} \times \mathbb{R}^{n} \rightarrow \mathbb{R}^{n},(i=1, \ldots, M)$ are continuous, and $g_{0}(\cdot, Y, a) \in L^{1}\left(\mathbb{R}^{+}\right)$.

(Hg2) $\exists k_{0}(\cdot) \in L^{\infty}\left(\mathbb{R}^{+} ; \mathbb{R}^{+}\right)$such that $\forall Y, Z \in \mathbb{R}^{n}, a \in A, \mathrm{t} \in \mathbb{R}^{+}$,

$$
\left|g_{0}(t, Y, a)-g_{0}(t, Z, a)\right|+\sum_{i=1}^{M}\left|g_{i}(t, Y)-g_{i}(t, Z)\right| \leq k_{0}(t)|Y-Z| .
$$

(Hg3) $\forall i \in\{1, \ldots, M\}, \exists L>0$ such that

$$
\left|g_{0}(t, Y, a)\right| \leq L,\left|g_{i}(t, Y)\right| \leq L, \forall Y \in \mathbb{R}^{n}, a \in A, t \in \mathbb{R}^{+} .
$$

Following [7], we introduce the canonical graph completion for the measure $\mu=\left(\mu_{1}, \ldots, \mu_{M}\right)$. Let $B$ be the left continuous primitive of $\mu$, i.e. $B \in B V\left([0, T] ; \mathbb{R}^{n}\right)$ and its distributional derivative $\dot{B}$ coincides with $\mu$ on $[0, T)$. In all the sequel, we will denote by $\mathcal{T}$ the set of all the discontinuity points of $B$ :

$$
\mathcal{T}:=\left\{t_{i}, i \in \mathbb{N}\right\}
$$

Furthermore, we introduce the functions:

$$
\psi_{t_{i}}(\sigma):=B\left(t_{i}^{-}\right)+\sigma\left(B\left(t_{i}^{+}\right)-B\left(t_{i}^{-}\right)\right), \text {for } \sigma \in[0,1], i \in \mathbb{N} \text {. }
$$


We will denote by $\xi$ the solution of:

$$
\frac{d \xi(\sigma)}{d \sigma}=\sum_{j=1}^{M} g_{j}(\sigma, \xi(\sigma)) \frac{d \psi_{t}^{j}(\sigma)}{d \sigma}, \text { for } \sigma \in(0,1], \xi(0)=\bar{\xi}
$$

and we set $\xi\left(\bar{\xi}, \psi_{t}\right):=\xi(1)-\bar{\xi}$. Now we are ready to state the definition of solution introduced by Dal Maso and Rampazzo in [7].

Definition 2.1 Fix an initial position and time $(X, \tau)$ and control variable a $\in \mathcal{A}$, the function $Y:=Y_{X, \tau}^{a} \in B V\left([\tau, T] ; \mathbb{R}^{n}\right)$ is a solution of (1.1) if for each Borel subset $\mathcal{B}$ of $] \tau, T[$ we have

$$
\begin{aligned}
\int_{\mathcal{B}} d Y(t) & =\int_{\mathcal{B}} g_{0}(t, Y(t), a(t)) d t \\
& +\sum_{i=1}^{M} \int_{\mathcal{B} \backslash \mathcal{T}} g_{i}(t, Y(t)) d \mu_{i}+\sum_{t_{i} \in \mathcal{B} \cap \mathcal{T}} \xi\left(Y\left(t_{i}^{-}\right), \psi_{t_{i}}\right),
\end{aligned}
$$

and $Y\left(\tau^{-}\right)=X$. Moreover, if $\tau \in \mathcal{T}$ we have $Y\left(\tau^{+}\right)=\xi\left(X, \psi_{\tau}\right)$.

Remark 2.2 In the case when $g_{i}(t, Y)=c_{i} \in \mathbb{R}, \mu_{i}=\delta_{t_{i}}$ (Dirac measure at $t_{i}$ ) for $i=1, \ldots, M$, we have $\xi\left(Y\left(t_{i}^{-}\right), \psi_{t_{i}}\right)=c_{i}$.

We now use the graph completion technique to construct a reparameterized system which has a unique absolutely continuous solution. In order to do that, we define $\mathcal{W}:[0, T] \rightarrow[0,1]$ as follows:

$$
\mathcal{W}(t)=\frac{t+V_{0}^{t}(B)}{T+V_{0}^{T}(B)}, \text { for } t \in[0, T]
$$

then $\mathcal{W}$ is continuous on $[0, T] \backslash \mathcal{T}$. The canonical graph completion of $B$ corresponding to the family of linear functions $\left(\psi_{t}\right)_{t \in \mathcal{T}}$ is then defined by:

$$
\begin{aligned}
& \Phi(s)=\left(\phi^{0} ; \phi^{1}, \ldots, \phi^{M}\right)(s) \\
& = \begin{cases}(t ; B(t)) & \text { if } s=\mathcal{W}(t), t \in[0, T] \backslash\left\{t_{1}, \ldots, t_{M}\right\} \\
\left(t_{i} ; \psi_{t_{i}}\left(\frac{s-\mathcal{W}\left(t_{i}\right)}{[\mathcal{W}]_{t_{i}}}\right)\right) & \text { if } s \in\left[\mathcal{W}\left(t_{i}\right), \mathcal{W}\left(t_{i}^{+}\right)\right], i \in \mathcal{T}\end{cases}
\end{aligned}
$$

For any control $a \in \mathcal{A}$, any initial data $X \in \mathcal{K}$, and any $\sigma \in(0,1)$, we denote by $Z_{X, \sigma}^{a}:[\sigma, 1] \rightarrow \mathbb{R}^{n}$ the solution of

$$
\left\{\begin{aligned}
\frac{d Z}{d s}(s) & =g_{0}\left(\phi^{0}(s), Z(s), a\left(\phi^{0}(s)\right)\right) \frac{d \phi^{0}(s)(s)}{d s} \\
& +\sum_{i=1}^{M} g_{i}\left(\phi^{0}(s), Z(s)\right)\left(\mu_{i}^{a}\left(\phi^{0}(s)\right) \frac{d \phi^{0}(s)(s)}{d s}+\frac{d \phi^{i}(s)}{d s}\right) \quad \text { for } s \in(\sigma, 1], \\
Z(\sigma) & =X,
\end{aligned}\right.
$$

where $\mu^{a}$ is the absolutely continuous part of the measure $\mu$ with respect to the Lebesgue measure, i.e. $\mu(t)=\mu^{a}(t) d t+\mu^{s}$.

Theorem 2.3 Assume (Hco) and (Hg1)-(Hg3), then $Y_{X, \tau}^{a} \in B V\left([\tau, T] ; \mathbb{R}^{n}\right)$ is a solution of (1.1) if and only if there exists a solution $Z_{X, \sigma}^{a} \in A C\left([\sigma, 1] ; \mathbb{R}^{n}\right)$ of $(2.6)$ corresponding to the graph completion $\Phi$ defined in (2.5) such that

$$
Z_{X, \sigma}^{a}(\mathcal{W}(t))=Y_{X, \tau}^{a}(t), \quad \forall t \in[\tau, T]
$$

where $\sigma=\mathcal{W}(\tau)$, and $\mathcal{W}$ is given by $(2.4)$. 
Proof. It is the same proof as in [[6], Theorem 2.2] by adapting the proof given for $M=N=1$ in [[3], Theorem 2.8].

Remark 2.4 We point out that the definition of the reparameterized system depends on the family $\left(\psi_{t}\right)_{t \in \mathcal{T}}$, unless the vector fields satisfy some commutative assumptions. However, in this paper, the dependency on the choice of $\psi_{t}$ does not imply any specific difficulty in the sequel. We chose here to use the linear reparameterization in order to simply the presentation of the main ideas.

\subsection{State constrained problems}

Now we consider the same problem with state constraints. The equality (2.7) always holds true, but here the problem is to make sure that the reparameterization of a state constrained solution also satisfies the state constraints. Consider a set of state constraints $\mathcal{K}$ defined by:

$$
\mathcal{K}=\bigcap_{j=1}^{l}\left\{x, h_{j}(x) \leq 0\right\}, \stackrel{\circ}{\mathcal{K}}=\bigcap_{j=1}^{l}\left\{x, h_{j}(x)<0\right\}
$$

for a finite family of $C^{1,1}$ functions $\left\{h_{j}: \mathbb{R}^{n} \rightarrow \mathbb{R}\right\}_{j=1, \ldots, l}$. We also denote by

$$
I(x)=\left\{j \in\{1, \ldots, l\}, h_{j}(x)=0\right\}
$$

the active set of index values at a point $x \in \partial \mathcal{K}$.

We say that a trajectory $Y_{X, \tau}^{a}$, solution of (1.1), is admissible if for every $t \in[\tau, T], Y_{X, \tau}^{a}(t) \in \mathcal{K}$. Here in order to make sure that the "fictive" part of the trajectories of reparameterized system satisfies the state constraints, we need to consider the following viability condition: $\forall t \geq 0, \forall Y \in \partial \mathcal{K}, \forall j \in$ $I(Y), \forall i=1, \ldots, M$,

$$
g_{i}(t, Y) \cdot \nabla_{x} h_{j}(Y) \leq 0 .
$$

We then have the following theorem:

Theorem 2.5 Assume that the assumption of Theorem 2.3 are satisfied and that the viability condition (2.9) holds. Then $Y_{X, \tau}^{a}(t) \in \mathcal{K}$ for every $t \in[\tau, T]$ if and only if $Z_{X, \sigma}^{a}(s) \in \mathcal{K}$ for every $s \in[\mathcal{W}(\tau), 1]$.

Proof. Assume $Z(\cdot) \in \mathcal{K}$. Since we have $Y(\cdot)=Z(\mathcal{W}(\cdot))$, and for each $t \in[\tau, T], \mathcal{W}(t) \in[\sigma, 1]$, then we get $Z(\mathcal{W}(t)) \in \mathcal{K}$ by the definition of $Z(\cdot)$, which implies

$$
Y(t) \in \mathcal{K}, \forall t \in[\tau, T] .
$$

If $Y(\cdot) \in \mathcal{K}$, let us prove that $Z(s) \in \mathcal{K}$ for each $s \in[\sigma, 1]$, where

$$
Z(s)= \begin{cases}Y(t), & \text { if } s=\mathcal{W}(t), t \in[0, T] \backslash \mathcal{T} \\ \exp \left[\left(s-\mathcal{W}\left(t_{i}\right)\right) \frac{[B]_{t_{i}}}{[\mathcal{W}]_{t_{i}}} g_{i}\right] Y\left(t_{i}\right), & \text { if } s \in\left[\mathcal{W}\left(t_{i}\right), \mathcal{W}\left(t_{i}^{+}\right)\right], i=1, \ldots, M,\end{cases}
$$

If $t \in[0, T] \backslash \mathcal{T}$ s.t. $s=\mathcal{W}(t)$, we have

$$
Z(s)=Y(t) \in \mathcal{K} .
$$

On the other side, if $\exists t_{i} \in \mathcal{T}$ s.t. $s \in\left[\mathcal{W}\left(t_{i}\right), \mathcal{W}\left(t_{i}^{+}\right)\right]$, we have $\left.Z(s)=\exp \left[(s-\mathcal{W}(t)) \frac{[B]_{t_{i}}}{[\mathcal{W}]_{t_{i}}} g_{i}\right] Y\left(t_{i}\right)\right)$. As $Y\left(t_{i}\right) \in \mathcal{K}$, by the boundary condition $(2.9)$, the dynamics $\frac{[B]_{t_{i}}}{[\mathcal{W}]_{t_{i}}} g_{i}(s, Z)$ is a contingent direction to $\mathcal{K}$ at every $Z \in \mathcal{K}$. Therefore, by using Viability Theorem 3.3.5 in [1], we get that

$$
Z(s) \in \mathcal{K}, \text { for } s \in\left[\mathcal{W}\left(t_{i}\right), \mathcal{W}\left(t_{i}^{+}\right)\right] .
$$


Remark 2.6 In case a more general family of Lipschitz continuous maps for $\left\{\psi_{t}\right\}_{t \in \mathcal{T}}$ is considered, these maps should be increasing, i.e.

$$
\psi_{t}^{\prime}(\sigma) \geq 0, \forall \sigma \in[0,1]
$$

in order to ensure the viability property (2.9).

\subsection{The control problem}

Let $\varphi: \mathbb{R}^{n} \rightarrow \mathbb{R}$ be a given bounded uniformly continuous function and $T$ be a final time. Our aim is to characterize the following value function

$$
v(\tau, X):=\inf \left\{\varphi\left(Y_{X, \tau}^{a}(T)\right) \mid a \in \mathcal{A}, Y_{X, \tau}^{a}(t) \in \mathcal{K} \text { on }[\tau, T]\right\}
$$

where $Y_{X, \tau}^{a}(\cdot)$ is the solution of equation (1.1).

Formally, we derive an HJB equation satisfied by $v$ :

$$
\begin{cases}-v_{t}(t, X)+H(t, X, D v(t, X))=0 & \text { for }(t, X) \in(0, T) \times \mathcal{K}, \\ v(T, X)=\varphi(X) & \text { for } X \in \mathcal{K}\end{cases}
$$

where the Hamiltonian is

$$
H(t, X, p)=\sup _{a \in \mathcal{A}}\left\{-p \cdot\left(g_{0}(t, X, a)+\sum_{i=1}^{M} g_{i}(t, X) \mu_{i}\right)\right\} .
$$

The problem here is that the product $D v \cdot \mu_{i}$ is not well defined, since $\mu_{i}$ is a measure and $v$ is not differentiable. In view of Theorem 2.3 , it is then natural to consider the auxiliary control problem governed by trajectories $Z_{X, \sigma}^{a}$ solutions of the reparameterized system 2.6. Then the corresponding value function is defined as follows:

$$
\bar{v}(\sigma, X)=\inf _{a \in \mathcal{A}}\left\{\varphi\left(Z_{X, \sigma}^{a}(1)\right), Z_{X, \sigma}^{a}(s) \in \mathcal{K} \text { on }[\sigma, 1]\right\} .
$$

Theorem 2.7 Let $v$ and $\bar{v}$ be defined respectively by (2.11) and (2.14). For each $X \in \mathcal{K}$ and $\tau \in[0, T]$, we have

$$
v(\tau, X)=\bar{v}(\mathcal{W}(\tau), X)
$$

where $\mathcal{W}$ is given by $(2.4)$.

Proof. By Theorem 2.3 we have

$$
Y_{X, \tau}^{a}(T)=Z_{X, \mathcal{W}(\tau)}^{a}(\mathcal{W}(T))=Z_{X, \sigma}^{a}(1),
$$

then (2.15) holds by the definition of $v$ and $\bar{v}$.

According to this theorem, we can turn our attention to the HJB equation for the function $\bar{v}$ to avoid dealing with the Radon measures in the dynamics.

The dynamic programming principle satisfied by $\bar{v}$ leads to the following HJB equation:

$$
\begin{cases}-\bar{v}_{s}(s, X)+H(s, X, D \bar{v}(s, X))=0 & (s, X) \in(0,1) \times \mathcal{K}, \\ \bar{v}(1, X)=\varphi(X) & \text { for } X \in \mathcal{K}\end{cases}
$$

where the Hamiltonian is

$$
\begin{aligned}
H(s, X, p)= & \sup _{a \in \mathcal{A}}\left\{-p \cdot\left(g_{0}\left(\phi^{0}(s), Z(s), a\left(\phi^{0}(s)\right)\right) \frac{d \phi^{0}(s)}{d s}\right)\right. \\
& \left.+\sum_{i=1}^{M} g_{i}\left(\phi^{0}(s), Z(s)\right)\left(\mu_{i}^{a}\left(\phi^{0}(s)\right) \frac{d \phi^{0}(s)(s)}{d s}+\frac{d \phi^{i}(s)}{d s}\right)\right\} .
\end{aligned}
$$

Note that $\mathcal{K}$ is a closed set. Moreover, the derivatives of $\phi^{0}$ and $\phi^{i}$ are just measurable functions, we should first make precise the definition of the constrained $L^{1}$-viscosity solution of (2.16). 


\section{State constrained optimal control problems with measurable time-dependent dynamics}

In this section, we introduce the definition of viscosity solution for the HJB equation with a time measurable Hamiltonian and state constraints. To simplify the presentation, we set

$$
f(t, y, a)=g_{0}\left(\phi^{0}(t), y, a\right) \frac{d \phi^{0}(t)}{d t}+\sum_{i=1}^{M} g_{i}\left(\phi^{0}(t), y\right)\left(\mu_{i}^{a}\left(\phi^{0}(t)\right) \frac{d \phi^{0}(t)}{d t}+\frac{d \phi^{i}(t)}{d t}\right)
$$

which is measurable in $t$, Lipschitz continuous in $y$ and continuous in $a$. Equation (2.6) then read

$$
\left\{\begin{array}{l}
\dot{y}(t)=f(t, y(t), a(t)), \quad \text { for } t \in(\tau, 1) \\
y(\tau)=x
\end{array}\right.
$$

Remark 3.1 Let us recall that under assumptions (Hco), (Hg1)-(Hg3), for every a $\in \mathcal{A}$, the differential equation (3.19) admits an absolutely continuous solution.

Let $\mathcal{K}$ be the closed subset of $\mathbb{R}^{n}$ defined in (2.8) which is an intersection of finite smooth manifolds. For each initial time and position $(\tau, x) \in[0,1) \times \mathcal{K}$, we define the set of admissible trajectories by

$$
S_{[\tau, 1]}^{\mathcal{K}}(x):=\left\{y_{x, \tau}^{a} \text { solution of }(3.19), y_{x, \tau}^{a}(s) \in \mathcal{K} \text { for } s \in[\tau, 1]\right\} .
$$

Let $\varphi: \mathbb{R}^{n} \rightarrow \mathbb{R}$ be a given function satisfying:

(Hid) The function $\varphi: \mathbb{R}^{n} \rightarrow \mathbb{R}$ is uniformly continuous and bounded.

The optimal value function $\vartheta: \mathbb{R}^{+} \times \mathbb{R}^{n} \rightarrow \mathbb{R}$ associated to this problem is defined by:

$$
\vartheta(\tau, x):=\inf \left\{\varphi\left(y_{x, \tau}^{a}(1)\right), y_{x, \tau}^{a} \in S_{[\tau, 1]}^{\mathcal{K}}(x)\right\}
$$

Remark 3.2 We adopt the convention $\vartheta(\tau, x)=\|\varphi\|_{L^{\infty}(\mathcal{K})}+1$, when the set of admissible trajectories is empty: $S_{[\tau, 1]}^{\mathcal{K}}(x)=\emptyset$. Of course this value can be replaced by any other constant bigger than $\|\varphi\|_{L^{\infty}(\mathcal{K})}$, and eventually by $+\infty$. But we need to take a finite constant in order to deal with finite valued functions.

The following boundary condition "Inward Pointing Condition", introduced by Soner [12] will be considered

(HK) $\exists \beta>0, \forall t \in[0,1], y \in \partial \mathcal{K}, \forall j \in I(y)$

$$
\exists a \in \mathcal{A} \text { s.t. } f(t, y, a) \cdot \nabla_{x} h_{j}(y)<-\beta .
$$

Remark 3.3 If $g_{0}$ satisfies (3.21) and $g_{i}$ satisfies (2.9), then the field $f$ defined in (3.18) satisfies the assumption (HK) for some $\beta>0$.

Our first aim is to characterize the function $\vartheta$ in $(3.20)$ as the unique $L^{1}$ viscosity solution (see the definition below) of the following HJB equation:

$$
\begin{cases}-u_{t}(t, x)+H(t, x, D u(t, x))=0 & \text { for }(t, x) \in(0,1) \times \mathcal{K}, \\ u(1, x)=\varphi(x) & x \in \mathcal{K}\end{cases}
$$

where the Hamiltonian is

$$
H(t, x, p)=\sup _{a \in \mathcal{A}}\{-p \cdot f(t, x, a)\} .
$$




\subsection{Uniform continuity of the value function}

We recall the dynamic programming principle for $\vartheta(\tau, X)$ :

Proposition 3.4 Assume (Hco), (Hg1)-(Hg3), (Hid) and (HK). Then the value function $\vartheta$ satisfies the following:

i) for all $x \in \mathcal{K}$,

$$
\vartheta(1, x)=\varphi(x)
$$

ii) Dynamic programming principle: for all $x \in \mathcal{K}, \tau \in[0,1]$ and $h \in[0,1-\tau]$, we have:

$$
\vartheta(\tau, x)=\inf _{y_{x, \tau}^{a} \in S_{[\tau, 1]}^{\mathcal{K}}(x)} \vartheta\left(\tau+h, y_{x, \tau}^{a}(\tau+h)\right),
$$

We will prove the continuity of the value function on $(0,1) \times \mathcal{K}$.

Remark 3.5 From [9, Theorem 3.1], we note that for any $x_{0} \in \stackrel{\circ}{\mathcal{K}}$, there exists an admissible trajectory $x$ on $\left[t_{0}, 1\right]$ such that

$$
x\left(t_{0}\right)=x_{0}, x(t) \in \stackrel{\circ}{\mathcal{K}}, \forall t \in\left[t_{0}, 1\right] .
$$

In fact, there exists a small enough $\varepsilon>0$ such that

$$
\begin{gathered}
x_{0} \in \mathcal{K}_{\varepsilon}:=\bigcap_{j=1}^{r}\left\{x, h_{j}(x)+\varepsilon \leq 0\right\}, \\
\min _{\nu \in f(t, x, A)} \max _{j \in I(x)} \nabla h_{j}(x) \cdot \nu<-\frac{\alpha}{2}, x \in \partial \mathcal{K}_{\varepsilon}, t \in[0,1],
\end{gathered}
$$

by the continuity of $\nabla h_{j}$ and $x \rightarrow f(t, x, A)$. Then by this theorem there exists an admissible trajectory contained in $\mathcal{K}_{\varepsilon}$ which is in $\stackrel{\circ}{\mathcal{K}}$.

Proposition 3.6 Assume (Hco), (Hg1)-(Hg3), (Hid) and $(H K)$, the value function $\vartheta(\cdot, \cdot)$ is continuous on $(0,1) \times \stackrel{\circ}{\mathcal{K}}$.

In order to prove the continuity of the value function on the boundary, we use the relaxation method. The following proposition is a result of relaxation of state constraints:

Proposition 3.7 Assume (Hco), (Hg1)-(Hg3), (Hid) and (HK). Consider $\left(\mathcal{K}^{\varepsilon}\right)_{\varepsilon>0}$ a sequence of subsets of $\mathbb{R}^{n}$ such that

$$
\mathcal{K}^{\varepsilon}=\bigcap_{j=1}^{r}\left\{x, h_{j}(x)-\varepsilon \leq 0\right\},
$$

and we denote by $\vartheta_{\varepsilon}$ the value function associated to the control problem (3.20) with state constraints in $\mathcal{K}^{\varepsilon}$ (instead of $\mathcal{K}$ ). Then

$$
\lim _{\varepsilon \rightarrow 0} \vartheta_{\varepsilon}(t, x)=\vartheta(t, x) \text { uniformly on }(0,1) \times \mathcal{K} .
$$

Proof. By the definition of $\mathcal{K}^{\varepsilon}$, for every $x \in \mathcal{K}$, every $\varepsilon>0$ and $\eta \in(0, \varepsilon)$ we have

$$
\mathcal{K} \subset \mathcal{K}^{\eta} \subset \stackrel{\circ}{\mathcal{K}}, \lim _{\varepsilon \rightarrow 0} d\left(x, \mathbb{R}^{n} \backslash \mathcal{K}^{\varepsilon}\right)=0,
$$

then, for $t \in(0,1)$ and $x \in \mathcal{K}$ given, we have

$$
\vartheta_{\varepsilon}(t, x) \leq \vartheta(t, x)
$$


Let us set $l:=\lim _{\inf _{\varepsilon \rightarrow 0}} \vartheta_{\varepsilon}(t, x)$. For $k \in \mathbb{N}$ large enough, $\exists \varepsilon_{k}>0$ such that $\varepsilon_{k} \rightarrow 0$ and

$$
\vartheta_{\varepsilon_{k}}(t, x) \leq l+\frac{1}{2 k},
$$

then by the definition of the value function $\vartheta_{\varepsilon_{k}}$, there exists a trajectory $y_{x, t}^{\varepsilon_{k}} \in S_{[t, 1]}^{\mathcal{K}_{k}}(x)$ such that

$$
\varphi\left(y_{x, t}^{\varepsilon_{k}}(1)\right) \leq \vartheta_{\varepsilon_{k}}(t, x)+\frac{1}{2 k} \leq l+\frac{1}{k} .
$$

By remark 3.1, we can extract from $y_{x, t}^{\varepsilon_{k}}$ a convergent subsequence towards some trajectory $y_{x, t} \in$ $S_{[t, 1]}^{\mathcal{K}^{\varepsilon} k}(x)$ for every $k>0$. We then obtain that $y_{x, t} \in S_{[t, 1]}^{\mathcal{K}}(x)$ by using $(3.25)$. Let $k$ tend to $+\infty$ in (3.27) and use the fact that $\varphi$ is continuous, we prove that

$$
\varphi\left(y_{x, t}(1)\right) \leq l .
$$

Then we have

$$
\vartheta(t, x) \leq \varphi\left(y_{x, t}(1)\right) \leq l=\lim \inf _{\varepsilon \rightarrow 0} \vartheta_{\varepsilon}(t, x) .
$$

Combining (3.26) and (3.28), we get that

$$
\vartheta(t, x) \leq \lim \inf _{\varepsilon \rightarrow 0} \vartheta_{\varepsilon}(t, x) \leq \lim \sup _{\varepsilon \rightarrow 0} \vartheta_{\varepsilon}(t, x) \leq \vartheta(t, x)
$$

which implies $\lim _{\varepsilon \rightarrow 0} \vartheta_{\varepsilon}(t, x)=\vartheta(t, v)$.

For each $\varepsilon>0$, let $y_{x, t}^{\varepsilon} \in S_{[t, 1]}^{\mathcal{K}^{\varepsilon}}(x)$ such that $\vartheta_{\varepsilon}(t, x)=\varphi\left(y_{x, t}^{\varepsilon}(1)\right)$. By [9, Theorem 3.1], there exist a $\hat{y}_{x, t} \in S_{[t, 1]}^{\mathcal{K}}(x)$ and a constant $K$ such that

$$
\left\|\hat{y}_{x, t}-y_{x, t}^{\varepsilon}\right\|_{W^{1,1}\left([t, 1] ; \mathbb{R}^{n}\right)} \leq K \varepsilon .
$$

Then we have

$$
\begin{array}{r}
0 \leq \vartheta(t, x)-\vartheta_{\varepsilon}(t, x) \leq \varphi\left(\hat{y}_{x, t}(1)\right)-\varphi\left(y_{x, t}^{\varepsilon}(1)\right) \\
\leq m_{\varphi}\left\|\hat{y}_{x, t}-y_{x, t}^{\varepsilon}\right\|_{W^{1,1}} \leq m_{\varphi} K \varepsilon
\end{array}
$$

where $m_{\varphi}(\cdot)$ is the Lipshitz constant of $\varphi$. Let $\varepsilon \rightarrow 0$, we get that $\vartheta_{\varepsilon} \rightarrow \vartheta$ uniformly on $(0,1) \times \mathcal{K}$.

Now we get the theorem:

Theorem 3.8 Assume (Hco) (Hg1)-(Hg3) (Hid) and (HK), the value function $\vartheta(\cdot, \cdot)$ is uniformly continuous and bounded on $(0,1) \times \mathcal{K}$.

Proof. We only need to prove that $\forall \tau \in(0,1), \vartheta(\tau, \cdot)$ is continuous on $\mathcal{K}$. For every $\tau \in(0,1)$ and every $x \in \mathcal{K}$, define $\mathcal{K}^{\varepsilon}$ and $\vartheta_{\varepsilon}$ as in proposition 3.7 . Then we have

$$
\vartheta(\tau, x)=\lim _{\varepsilon \rightarrow 0} \vartheta_{\varepsilon}(\tau, x) \text { uniformly on }(0,1) \times \mathcal{K} .
$$

According to (HK), by the continuity of $\nabla h_{j}(x)$ and $f(t, x, p)$ on $x$, for small $\varepsilon$, we have $\forall t \in[0,1], y \in$ $\partial \mathcal{K}^{\varepsilon}, j \in I(y)$,

$$
\exists a \in \mathcal{A} \text { s.t. } f(t, y, a) \cdot \nabla_{x} h_{j}(y)<-\frac{\beta}{2} .
$$

Using (3.25) and proposition 3.6, we get that $\vartheta_{\varepsilon}(\tau, \cdot)$ is continuous on $\mathcal{K} \subset \mathcal{K}^{\varepsilon}$ and bounded, then by the uniform convergence of $\vartheta_{\varepsilon}$, the limit $\vartheta(\tau, \cdot)$ is continuous on $\mathcal{K}$.

Then $\forall \tau \in(0,1), \forall x, z \in \mathcal{K}$, without loss of generality we suppose that $\vartheta(\tau, x) \geq \vartheta(\tau, z)$. Let $y_{z, \tau}^{a} \in S_{[\tau, 1]}^{\mathcal{K}}$ such that $\vartheta(\tau, z)=y_{z, \tau}^{a}(1)$, then we have

$$
0 \leq \vartheta(\tau, x)-\vartheta(\tau, z) \leq \varphi\left(y_{x, \tau}^{a}(1)\right)-\varphi\left(y_{z, \tau}^{a}(1)\right)
$$

Then by the uniform continuity of $\varphi$ and Gronwall lemma, we deduce the uniform continuity of $\vartheta$. Finally, since $\varphi$ is bounded, we obtain that $\vartheta$ is bounded on $(0,1) \times \mathcal{K}$. 


\subsection{Definition of $L^{1}$-viscosity solutions of HJB equations}

This section is devoted to the definition of the $L^{1}$-viscosity solutions of the HJB equation (3.22) and the characterization of the value function $\vartheta$. The following definition can be seen as the combination of the definition of $L^{1}$-viscosity solutions for the HJB equations with a time measurable Hamiltonian introduced in $[11,10,6]$ and the definition of constrained viscosity solutions introduced in Soner [12].

Definition 3.9 ( $L^{1}$-viscosity solution) Let $u:(0,1] \times \mathcal{K} \rightarrow \mathbb{R}$ be a bounded Lipschitz continuous function.

- We say that $u$ is a $L^{1}$-viscosity super-solution if $\forall b \in L^{1}(0,1), \phi \in C^{1}\left(\mathbb{R}^{n}\right)$ and $\left(t_{0}, x_{0}\right) \in$ $(0,1) \times \mathcal{K}$ local minimum point of $u(t, x)-\int_{0}^{t} b(s) d s-\phi(x)$, we have

$$
\lim _{\delta \rightarrow 0^{+}} \operatorname{esssup}_{\left|t-t_{0}\right| \leq \delta} \sup _{\substack{\left.\mid x_{0}, \delta\right) \cap \mathcal{K}, p \in B\left(D \phi\left(x_{0}\right), \delta\right)}}\{H(t, x, p)-b(t)\} \geq 0 .
$$

- We say that $u$ is a $L^{1}$-viscosity sub-solution if $\forall b \in L^{1}(0,1), \phi \in C^{1}\left(\mathbb{R}^{n}\right)$ and $\left(t_{0}, x_{0}\right) \in(0,1) \times \stackrel{\circ}{\mathcal{K}}$ local maximum point of $u(t, x)-\int_{0}^{t} b(s) d s-\phi(x)$, we have

$$
\lim _{\delta \rightarrow 0^{+}} \operatorname{essinf}_{\left|t-t_{0}\right| \leq \delta} \inf _{x \in B\left(x_{0}, \delta\right), p \in B\left(D \phi\left(x_{0}\right), \delta\right)}\{H(t, x, p)-b(t)\} \leq 0 .
$$

- We say that $u$ is a $L^{1}$-viscosity solution if it is both a $L^{1}$-viscosity super-solution and a $L^{1}$ viscosity sub-solution and the final condition is satisfied:

$$
u(1, x)=\varphi(x) \text { in } \mathcal{K} .
$$

Remark 3.10 In fact, there are many more formulations. For example we may replace $\phi \in C^{1}$ by $\phi \in C^{2}, C^{\infty}, \ldots$ We may also replace local maximum by global, or local strict, or global strict. We can also give another equivalent formulation of definition by generalizing the definition introduced by Ishii [10] to a closed subset $\mathcal{K}$. For more details, see Lions and Perthame [11].

Remark 3.11 If the Hamiltonian $H$ is continuous, this definition is the same notion of viscosity solutions introduced in Soner [12].

Theorem 3.12 Suppose (Hco), (Hg1)-(Hg3), (Hid), (HK) hold. Then the value function $\vartheta$ is a $L^{1}$-viscosity solution of (3.22).

Proof. We first prove that $\vartheta$ is a $L^{1}$-viscosity super-solution. Let $b \in L^{1}(0,1), \phi \in C^{1}\left(\mathbb{R}^{n}\right)$ and $\left(t_{0}, x_{0}\right) \in(0,1) \times \mathcal{K}$ local minimum point of $\vartheta(t, x)-\int_{0}^{t} b(s) d s-\phi(x)$. Without loss of generality, we suppose that

$$
\vartheta\left(t_{0}, x_{0}\right)-\int_{0}^{t_{0}} b(s) d s-\phi\left(x_{0}\right)=0
$$

then we have $\exists \delta>0$ small enough such that for $t \in\left[t_{0}-\delta, t_{0}+\delta\right], x \in B\left(x_{0}, \delta\right) \cap \mathcal{K}$,

$$
\vartheta(t, x)-\int_{0}^{t} b(s) d s-\phi(x) \geq 0 .
$$

By the DPP, $\forall \varepsilon>0, \exists a \in \mathcal{A}$ s.t. $\forall h \in\left[0,1-t_{0}\right]$,

$$
\vartheta\left(t_{0}+h, Y_{x_{0}, t_{0}}^{a}\left(t_{0}+h\right)\right) \leq \vartheta\left(t_{0}, x_{0}\right)+\varepsilon .
$$

Let $h$ small enough $(h \leq \delta)$, by $(3.31)$ we get

$$
\vartheta\left(t_{0}+h, Y_{x_{0}, t_{0}}^{a}\left(t_{0}+h\right)\right) \geq \int_{0}^{t_{0}+h} b(s) d s+\phi\left(Y_{x_{0}, t_{0}}^{a}\left(t_{0}+h\right)\right) .
$$


By (3.30), (3.32) and (3.33), we have

$$
\int_{0}^{t_{0}+h} b(s) d s+\phi\left(Y_{x_{0}, t_{0}}^{a}\left(t_{0}+h\right)\right) \leq \int_{0}^{t_{0}} b(s) d s+\phi\left(x_{0}\right)+\varepsilon,
$$

then

$$
\phi\left(x_{0}\right)-\phi\left(Y_{x_{0}, t_{0}}^{a}\left(t_{0}+h\right)\right)-\int_{t_{0}}^{t_{0}+h} b(s) d s+\varepsilon \geq 0,
$$

i.e.

$$
-\int_{t_{0}}^{t_{0}+h}\left[D \phi\left(Y_{x_{0}, t_{0}}^{a}(s)\right) \cdot f\left(s, Y_{x_{0}, t_{0}}^{a}(s), a\right)+b(s)\right] d s+\varepsilon \geq 0,
$$

then by the definition of the Hamiltonian, we have $\forall \varepsilon>0$

$$
\int_{t_{0}}^{t_{0}+h}\left[H\left(s, Y_{x_{0}, t_{0}}^{a}(s), D \phi\left(Y_{x_{0}, t_{0}}^{a}(s)\right)\right)-b(s)\right] d s+\varepsilon \geq 0,
$$

and we deduce that

$$
\int_{t_{0}}^{t_{0}+h}\left[H\left(s, Y_{x_{0}, t_{0}}^{a}(s), D \phi\left(Y_{x_{0}, t_{0}}^{a}(s)\right)\right)-b(s)\right] d s \geq 0 .
$$

By contradiction, if

$$
\lim _{\delta \rightarrow 0^{+}} \operatorname{esssup}_{\left|t-t_{0}\right| \leq \delta} \sup _{x \in B\left(x_{0}, \delta\right) \cap \mathcal{K}, p \in B\left(D \phi\left(x_{0}\right), \delta\right)}\{H(t, x, p)-b(t)\}<0,
$$

then $\exists \delta_{1}>0, E \subset\left[t_{0}-\delta_{1}, t_{0}+\delta_{1}\right]$ with $m(E)=0$ such that $\forall t \in\left[t_{0}-\delta_{1}, t_{0}+\delta_{1}\right] \backslash E, x \in B\left(x_{0}, \delta_{1}\right)$ and $p \in B\left(D \phi\left(x_{0}\right), \delta_{1}\right)$, we have $H(t, x, p)-b(t)<0$. By the continuity of $Y_{x_{0}, t_{0}}^{a}(\cdot), D \phi(\cdot)$ and $H(t, \cdot, \cdot)$, if $h$ is small enough, we get that for $s \in\left[t_{0}, t_{0}+h\right] \backslash E$,

$$
H\left(s, Y_{x_{0}, t_{0}}^{a}(s), D \phi\left(Y_{x_{0}, t_{0}}^{a}(s)\right)\right)-b(s)<0,
$$

which is a contradiction with (3.34).

By the similar arguments, we can prove that $\vartheta$ is a $L^{1}$-viscosity sub-solution. Finally, by the definition of $\vartheta$, we have $\vartheta(1, x)=\varphi(x)$ because $Y_{x, 1}^{a}(1)=x$.

\section{Uniqueness of the $L^{1}$ constrained viscosity solutions of HJB equations}

Consider the following Hamilton-Jacobi-Bellman equation

$$
\begin{cases}-u_{t}(t, x)+H(t, x, D u(t, x))=0 & \text { for }(t, x) \in(0,1) \times \mathcal{K}, \\ u(1, x)=\varphi(x) & x \in \mathcal{K} .\end{cases}
$$

We prove the comparison principle from which we can deduce the uniqueness of $L^{1}$-viscosity solution of $(3.22)$ on $\mathcal{K}$ satisfying the following property as in Soner [12]:

(A1) There exists positive constants $h, r$ and an $\mathbb{R}^{n}$-valued bounded, uniformly continuous map $\eta$ of $\mathcal{K}$ satisfying

$$
B(x+t \eta(x), r t) \subset \stackrel{\circ}{\mathcal{K}}, \forall x \in \mathcal{K} \text { and } t \in(0, h] .
$$

Theorem 4.1 (Comparison Principle) Assume that (Hco), (Hg1)-(Hg3), (Hid) and (A1) hold, let $u_{1}, u_{2}$ be two bounded uniformly continuous functions. Suppose that $u_{1}$ is a $L^{1}$-viscosity sub-solution of the HJB equation (3.22), $u_{2}$ is a $L^{1}$-viscosity super-solution of (3.22), and $u_{1}, u_{2}$ satisfy the final condition $u_{1}(1, x) \leq \varphi(x) \leq u_{2}(1, x)$ for every $x \in \mathcal{K}$. Then for every $t \in[0,1]$ and $x \in \mathcal{K}$, we have

$$
u_{1}(t, x) \leq u_{2}(t, x) .
$$


Proof. Let $v_{1}=u_{1} e^{t}$ and $v_{2}=u_{2} e^{t}$, then $v_{1}, v_{2}$ is respectively the $L^{1}$-viscosity sub-solution and $L^{1}$-viscosity super-solution of the HJB equation

$$
-v_{t}(t, x)+v(t, x)+H(t, x, D v(t, x))=0, \text { for } t \in(0,1), x \in \mathcal{K} .
$$

Let $\eta, r, h$ be as in the assumption (A1) in Soner [12], pick $\rho>0$ such that

$$
|\eta(x)-\eta(y)| \leq \frac{r}{2}, \forall x, y \in \mathcal{K} \text { and }|x-y|<\rho .
$$

We will prove that for all $(t, x) \in[0,1] \times \mathcal{K}$ by contradiction. Suppose that

$$
M:=\sup _{(t, x) \in[0,1] \times \mathcal{K}}\left\{v_{1}(t, x)-v_{2}(t, x)\right\}>0 .
$$

For all $\sigma \in(0, M)$, let $t_{\sigma} \in(0, M)$ and $z_{\sigma} \in \mathcal{K}$ such that

$$
v_{1}\left(t_{\sigma}, z_{\sigma}\right)-v_{2}\left(t_{\sigma}, z_{\sigma}\right) \geq M-\sigma>0 .
$$

The proof of the Theorem uses the classical techniques of doubling of variables and it is similar to the proof of Theorem 2.2 in Soner [12]. But here the function of doubling of variables should be adapted to the definition 3.9 which is new. We define $\Phi^{\varepsilon}:[0,1] \times[0,1] \times \mathcal{K} \times \mathcal{K} \rightarrow \mathbb{R}$ as follows:

$$
\begin{aligned}
\Phi^{\varepsilon}\left(t^{\prime}, s^{\prime}, x^{\prime}, y^{\prime}\right)= & v_{1} \quad\left(t^{\prime}, x^{\prime}\right)-v_{2}\left(s^{\prime}, y^{\prime}\right)-\left|\frac{x^{\prime}-y^{\prime}}{\varepsilon}-\frac{2}{r} \eta\left(z_{\sigma}\right)\right|^{2} \\
& -\left|\frac{y^{\prime}-z_{\sigma}}{\rho}\right|^{2}-\left|\frac{t^{\prime}-t_{\sigma}}{\nu}\right|^{2}-\left|\frac{t^{\prime}-s^{\prime}}{\alpha}\right|^{2} \\
& +\int_{0}^{t^{\prime}} b_{\epsilon}(\tau) d \tau+\int_{0}^{s^{\prime}} b_{\epsilon}(\tau) d \tau,
\end{aligned}
$$

where $b_{\epsilon}(\cdot) \in L^{1}\left(\mathbb{R}^{+}\right)$is positive and $b_{\epsilon} \rightarrow 0$ in $L^{1}\left(\mathbb{R}^{+}\right)$when $\epsilon \rightarrow 0$. Note that $z_{\sigma}+(2 \varepsilon / r) \eta\left(z_{\sigma}\right)$ is in $\mathcal{K}$ for small $\varepsilon$, we have

$$
\begin{gathered}
\Phi^{\varepsilon}\left(t_{0}, t_{0}, z_{\sigma}+\frac{2 \varepsilon}{r} \eta\left(z_{\sigma}\right), z_{\sigma}\right)=v_{1}\left(t_{0}, z_{\sigma}+\frac{2 \varepsilon}{r} \eta\left(z_{\sigma}\right)\right)-v_{2}\left(t_{0}, z_{\sigma}\right)+2 \int_{0}^{t_{0}} b_{\varepsilon}(\tau) d \tau \\
\geq v_{1}\left(t_{0}, z_{\sigma}\right)-v_{2}\left(t_{0}, z_{\sigma}\right)-\omega_{1}\left(c_{1} \varepsilon\right)+2 \int_{0}^{t_{0}} b_{\varepsilon}(\tau) d \tau,
\end{gathered}
$$

where $\omega_{1}(\cdot)$ is the modulus of continuity of $v_{1}\left(t_{0}, \cdot\right)$ in a neighborhood of $z_{\sigma}$ and $c_{1}$ is a positive constant. Let $\nu$ be small enough such that $\forall x, y \in \mathcal{K}, \Phi^{\varepsilon}(\cdot, \cdot, x, y)$ achieves its maximum at $\left(t_{0}, t_{0}\right)$. Suppose that $\Phi^{\varepsilon}$ achieves its maximum at $\left(t_{0}, t_{0}, x_{0}, y_{0}\right)$, we have

$$
\Phi^{\varepsilon}\left(t_{0}, t_{0}, x_{0}, y_{0}\right) \geq \Phi^{\varepsilon}\left(t_{0}, t_{0}, z_{\sigma}+\frac{2 \varepsilon}{r} \eta\left(z_{\sigma}\right), z_{\sigma}\right),
$$

then by (4.39) and (4.40), we obtain

$$
\begin{gathered}
\left|\frac{x_{0}-y_{0}}{\varepsilon}-\frac{2}{r} \eta\left(z_{\sigma}\right)\right|^{2}+\left|\frac{y_{0}-z_{\sigma}}{\rho}\right|^{2} \leq \omega_{1}\left(c_{1} \varepsilon\right)+\omega_{1}\left(\left|x_{0}-y_{0}\right|\right)+\sigma, \\
v_{1}\left(t_{0}, x_{0}\right)-v_{2}\left(t_{0}, y_{0}\right) \geq v_{1}\left(t_{0}, z_{\sigma}\right)-v_{2}\left(t_{0}, z_{\sigma}\right)-\omega_{1}\left(c_{1} \varepsilon\right) .
\end{gathered}
$$

Since $\omega_{1}$ is bounded, (4.42) yields that for small $\varepsilon, \rho$,

$$
\left|x_{0}-y_{0}\right| \leq c_{2} \varepsilon,\left|y_{0}-z_{\sigma}\right| \leq \rho, \omega_{1}\left(c_{1} \varepsilon\right)+\omega_{1}\left(c_{2} \varepsilon\right)+\sigma \leq 1,
$$

where $c_{2}$ is a positive constant. Then by (4.37) and (4.42) we obtain

$$
\left|\eta\left(y_{0}\right)-\eta\left(z_{\sigma}\right)\right| \leq \frac{r}{2},\left|\frac{x_{0}-y_{0}}{\varepsilon}-\frac{2}{r} \eta\left(z_{\sigma}\right)\right| \leq 1,
$$


combining these yields

$$
x_{0} \in B\left(y_{0}+\frac{2 \varepsilon}{r} \eta\left(z_{\sigma}\right), \varepsilon\right) \subset B\left(y_{0}+\frac{2 \varepsilon}{r} \eta\left(y_{0}\right), \frac{2 \varepsilon}{r} r\right) .
$$

Thus, (A1) implies $x_{0} \in \stackrel{\circ}{\mathcal{K}}$ for small $\varepsilon$. Now consider the maps:

$$
\begin{aligned}
\phi_{1}(x) & =v_{2}\left(t_{0}, y_{0}\right)+\left|\frac{x-y_{0}}{\varepsilon}-\frac{2}{r} \eta\left(z_{\sigma}\right)\right|^{2}+\left|\frac{y_{0}-z_{\sigma}}{\rho}\right|^{2}+\frac{t_{0}^{2}}{\nu^{2}}-\int_{0}^{t_{0}} b_{\varepsilon}(\tau) d \tau, \\
b_{1}(t) & =\frac{2}{\nu^{2}}\left(t-t_{0}\right)-b_{\varepsilon}(t), \\
\phi_{2}(y) & =v_{1}\left(t_{0}, x_{0}\right)-\left|\frac{x_{0}-y}{\varepsilon}-\frac{2}{r} \eta\left(z_{\sigma}\right)\right|^{2}-\left|\frac{y-z_{\sigma}}{\rho}\right|^{2}-\frac{t_{0}^{2}}{\nu^{2}}+\int_{0}^{t_{0}} b_{\varepsilon}(\tau) d \tau, \\
b_{1}(s) & =\frac{2}{\nu^{2}}\left(s-t_{0}\right)+b_{\varepsilon}(s) .
\end{aligned}
$$

Then $v_{1}(t, x)-\int_{0}^{t} b_{1}(\tau) d \tau-\phi_{1}(x)$ has a maximum at $\left(t_{0}, x_{0}\right) \in(0, T) \times \stackrel{\circ}{\mathcal{K}}$, and $v_{2}(s, y)-\int_{0}^{s} b_{2}(\tau) d \tau-$ $\phi_{2}(y)$ has a minimum at $\left(t_{0}, y_{0}\right) \in(0, T) \times \mathcal{K}$, by the definition of $L^{1}$-viscosity solutions we have

$$
\begin{gathered}
\lim _{\delta \rightarrow 0^{+}} \operatorname{essinf}_{\left|t-t_{0}\right| \leq \delta} \inf _{x \in B\left(x_{0}, \delta\right), p \in B\left(p_{\varepsilon}, \delta\right)}\left\{H(t, x, p)+v_{1}(t, x)-b_{1}(t)\right\} \leq 0, \\
\lim _{\delta \rightarrow 0^{+}} \operatorname{ess} \sup _{\left|s-t_{0}\right| \leq \delta} \sup _{y \in B\left(y_{0}, \delta\right) \cap \mathcal{K}, q \in B\left(p_{\varepsilon}+q_{\varepsilon}, \delta\right)}\left\{H(s, y, q)+v_{2}(s, y)-b_{2}(s)\right\} \geq 0,
\end{gathered}
$$

where $D \phi_{1}\left(x_{0}\right)=p_{\varepsilon}, D \phi_{2}\left(y_{0}\right)=p_{\varepsilon}+q_{\varepsilon}$ with

$$
p_{\varepsilon}=\frac{2}{\varepsilon}\left(\frac{x_{0}-y_{0}}{\varepsilon}-\frac{2}{r} \eta\left(z_{\sigma}\right)\right), q_{\varepsilon}=-2 \frac{y_{0}-z_{\sigma}}{\rho^{2}} .
$$

(4.42) yields that $\left|q_{\varepsilon}\right| \leq h(\varepsilon, \sigma)$, where $h(\cdot, \cdot)$ denotes a continuous positive function which has the value zero at the origin. For any $\varepsilon>0$, let $\zeta_{\varepsilon} \in C_{c}^{\infty}(\mathbb{R})$ be a standard mollifier, and we define $H_{\varepsilon}$ by $H_{\varepsilon}(\cdot, x, p)=\zeta_{\varepsilon} \star H(\cdot, x, p)$ for $(x, p) \in \mathcal{K} \times \mathbb{R}^{n}$. For small $\varepsilon, \rho, \sigma$, setting

$$
b_{\varepsilon}(t)=\sup _{x \in B\left(z_{\sigma}, 1\right), p \in B(0,2 / \varepsilon+1)}\left|H_{\varepsilon}(t, x, p)-H(t, x, p)\right|
$$

for $t \in(0, T)$, and we check that $b_{\varepsilon} \rightarrow 0$ in $L^{1}(0, T)$ as $\varepsilon \rightarrow 0$. Thus, we deduce from (4.47) and (4.48) that

$$
\begin{aligned}
& \lim _{\delta \rightarrow 0^{+}} \operatorname{essinf}_{\left|t-t_{0}\right| \leq \delta} \inf _{x \in B\left(x_{0}, \delta\right), p \in B\left(p_{\varepsilon}, \delta\right)}\left\{H_{\varepsilon}(t, x, p)+v_{1}(t, x)-\frac{2}{\nu^{2}}\left(t-t_{0}\right)\right\} \leq 0, \\
& \lim _{\delta \rightarrow 0^{+}} \operatorname{ess} \sup _{\left|s-t_{0}\right| \leq \delta} \sup _{y \in B\left(y_{0}, \delta\right) \cap \mathcal{K}, q \in B\left(p_{\varepsilon}+q_{\varepsilon}, \delta\right)}\left\{H_{\varepsilon}(s, y, q)+v_{2}(s, y)-\frac{2}{\nu^{2}}\left(s-t_{0}\right)\right\} \geq 0 .
\end{aligned}
$$

Then by the continuity of $H_{\varepsilon}, v_{1}$ and $v_{2},(4.50)$ and (4.51) are equivalent to

$$
\begin{gathered}
H_{\varepsilon}\left(t_{0}, x_{0}, p_{\varepsilon}\right)+v_{1}\left(t_{0}, x_{0}\right) \leq 0, \\
H_{\varepsilon}\left(t_{0}, y_{0}, p_{\varepsilon}+q_{\varepsilon}\right)+v_{2}\left(t_{0}, y_{0}\right) \geq 0 .
\end{gathered}
$$

Subtract (4.53) from (4.52),

$$
\begin{aligned}
v_{1}\left(t_{0}, x_{0}\right)-v_{2}\left(t_{0}, y_{0}\right) \leq & H_{\varepsilon}\left(t_{0}, y_{0}, p_{\varepsilon}+q_{\varepsilon}\right)-H_{\varepsilon}\left(t_{0}, x_{0}, p_{\varepsilon}\right) \\
\leq & H_{\varepsilon}\left(t_{0}, y_{0}, p_{\varepsilon}+q_{\varepsilon}\right)-H_{\varepsilon}\left(t_{0}, y_{0}, p_{\varepsilon}\right) \\
& +H_{\varepsilon}\left(t_{0}, y_{0}, p_{\varepsilon}\right)-H_{\varepsilon}\left(t_{0}, x_{0}, p_{\varepsilon}\right) \\
\leq & \zeta_{\varepsilon} \star m\left(t_{0}, h(\varepsilon, \sigma)+c_{2} \varepsilon\right),
\end{aligned}
$$


where $m(t, \cdot)$ is the continuous modulus of $H(t, \cdot, p)$. Using (4.39), (4.43) and (4.54) we have

$$
\begin{aligned}
& \max _{x \in \mathcal{K}}\left\{v_{1}\left(t_{0}, x\right)-v_{2}\left(t_{0}, x\right)\right\} \leq v_{1}\left(t_{0}, z_{\sigma}\right)-v_{2}\left(t_{0}, z_{\sigma}\right)+\sigma \\
& \leq v_{1}\left(t_{0}, x_{0}\right)-v_{2}\left(t_{0}, y_{0}\right)+\omega_{1}\left(c_{1} \varepsilon\right)+\sigma \\
& \leq \zeta_{\varepsilon} \star m\left(t_{0}, h(\varepsilon, \sigma)+c_{2} \varepsilon\right)+\omega_{1}\left(c_{1} \varepsilon\right)+\sigma .
\end{aligned}
$$

Now send first $\varepsilon$ then $\sigma$ to zero, we get

$$
\max _{x \in \mathcal{K}}\left\{v_{1}\left(t_{0}, x\right)-v_{2}\left(t_{0}, x\right)\right\} \leq 0 .
$$

As $u_{1}\left(t_{0}, x\right)=e^{t_{0}} v_{1}\left(t_{0}, x\right)$ and $u_{2}\left(t_{0}, x\right)=e^{t_{0}} v_{2}\left(t_{0}, x\right)$, we obtain

$$
\max _{x \in \mathcal{K}}\left\{u_{1}\left(t_{0}, x\right)-u_{2}\left(t_{0}, x\right)\right\} \leq 0, \forall t_{0} \in(0, T),
$$

which ends the proof.

\section{Conclusion}

In this paper, we have dealt with state-constrained optimal control problems of discontinuous trajectories by means of a reparameterized method. We have proved that the value function of the reparameterized system with state constraints is characterized as the unique $L^{1}$-viscosity solution of an Hamilton-Jacobi-Bellman equation with a time-measurable dependent Hamiltonian. However, there are still some interesting work that we could continue to investigate. We can consider a control system with control terms in the measure which is more complicated and can describe some real applications. The same problem with other kinds of boundary conditions is also interesting to investigate. Finally, the numerical simulations are also interesting and necessary.

\section{References}

[1] J.-P. Aubin (1991). Viability Theory, Birkhäuser, Boston, Basel, Berlin.

[2] G. Barles (2006). A new stability result for viscosity solutions of nonlinear parabolic equations with weak convergence in time, C.R. Acad. Sci. Paris, Ser. I, 343(3):173-78.

[3] A. Briani (1999). A Hamilton-Jacobi equation with measures arising in $\Gamma$-convergence of optimal control problems, Differential and Integral Equations, 12(6), pp. 849-886.

[4] O. Bokanowski, N. Forcadel and H. Zidani (2011). Deterministic state constrained optimal control problems without controllability assumptions, ESAIM: COCV, DOI: 10.1051/cocv/2010030.

[5] A. Bressan and F. Rampazzo (1988). On differential systems with vector-valued impulsive controls, Boll. Un. Mat. Ital, 7(2-B): 641-656.

[6] A. Briani and H. Zidani (2011). Characterisation of the value function of final state constrained control prolems with BV trajectories, Comm. Math. Appl. Anal, vol. 10(6), pp. 1567-1587.

[7] G. Dal Maso, and F. Rampazzo (1991). On systems of ordinary differential equations with measures as controls, Differential and Integral Equations, 4(4), pp. 738-765.

[8] H. Frankowska and S. Plaskacz (2010). Semicontinuous solutions of Hamilton-Jacobi-Bellman equations with degenerate state constraints, J. Math. Anal. Appl., 251 (2000), pp. 818-838. 
[9] H. Frankowska and R.B. Vinter (2000). Existence of neighboring feasible trajectories: applications to dynamic programming for state-constrained optimal control problems, J. Optim. Theory Appl., 104, pp. 21-40.

[10] H. Ishii (1985). Hamilton-Jacobi equations with discontinuous Hamiltonians on arbitrary open sets. I, Bull. Facul. Sci. Eng., 28:33-77.

[11] P.L. Lions and B. Perthame (1987). Remarks on Hamilton-Jacobi equations with measurable time-dependant Hamiltonians, Nonlinear analysis, Theory, Methods Applications, 11(5):613-612.

[12] H.M. Soner (1986). Optimal control with state-space constraint. I, SIAM J.Control Optim., 24(3), pp. 552-561. 